\title{
EVALUACIÓN DE ESTRATEGIAS EDUCATIVAS DE INTEGRACIÓN INTERCULTURAL
}

\section{EVALUATION OF EDUCATIONAL STRATEGIES FOR INTERCULTURAL INTEGRATION}

\author{
Alix Casadiego Cabrales ${ }^{1}$ \\ Leidy Carolina Cuervo
}

Alvaro Avendaño Rodríguez ${ }^{3}$

Karina Avendaño Casadiego ${ }^{4}$

Universidad Surcolombiana

Universidad del Tolima

\section{RESUMEN}

Los principios del Proyecto Educativo de la Universidad Surcolombiana proponen crear

$1 \quad$ Licenciada en Pedagogía Infantil- Universidad Surcolombiana, Mg. en educación Universidad Surcolombiana, Doctora en Sociedad del Conocimiento, Universidad Internacional de la Rioja. ORCID http://orcid.org/0000-00034376-5874 alix.casadiego@usco.edu.co, tel (057) 8754753, Universidad Surcolombiana, Neiva $(H)$, Colombia

2 Licenciada en Pedagogía Infantil- Universidad Surcolombiana, Mg. en educación Universidad Surcolombiana, Candidata a Doctor en Investigación y DocenciaUNADE-México, Vicerrectora de Investigación y Proyección ORCID https://orcid.org/0000-0002-6927-0776 carolina.cuervo@usco.edu.co,tel (057) 8645141 Universidad Surcolombiana, Neiva $(H)$, Colombia 3 Mg. Desarrollo Comunitario, Universidad Surcolombiana, Docente de planta, Universidad Surcolombiana alave@usco.edu.co, tel (057) 8754753

Universidad Surcolombiana, Neiva (H), Colombia ORCID https://orcid.org/0000-0003-0300-400X

4 Egresada en Biología, Universidad del Tolima, Mg. Biología Molecular y biotecnología, Universidad de Bangor, Reino Unido. ORCID https://orcid.org/0000-0002-30717737 kavendanoc@gmail.com, tel 3124212631 Universidad del Tolima, Ibagué ( $T$ ), Colombia las condiciones que contribuyan a una mejor convivencia en un ambiente multicultural. De acuerdo con ello, el objetivo de la presente investigación es evaluar una estrategia de integración propuesta por el programa Educación Infantil, con el fin de lograr un mayor acercamiento con ciudadanos de otros países y culturas. A través de cuestionarios se recogieron las percepciones de los estudiantes sobre la relación de estas actividades con el logro de los principios institucionales. Los resultados dejan ver que la actividad que más relacionan los estudiantes con la asimilación y comprensión de estos principios es la danza, mientras que el aprendizaje de una segunda lengua fue la actividad más débilmente asociada.

PALABRAS CLAVE: Multicultural, Interculturalidad, estudiantes, integración. 
EVALUATION OF EDUCATIONAL STRATEGIES OF INTERCULTURAL INTEGRATION

\section{SUMMARY}

The principles of the Educational Project of the Surcolombiana University, propose to create the conditions that contribute to a better coexistence in a multicultural environment. Accordingly, the objective of this research is to evaluate an integration strategy proposed by the Infant Education program, in order to achieve a closer relationship with citizens of other countries and cultures. Through questionnaires, students' perceptions of the relationship between these activities and the achievement of institutional principles were collected. The results show that the activity that students most relate to the assimilation and understanding of these principles is dance, while learning a second language was the most weakly associated activity.

KEYWORDS: Multicultural, Interculturality, students, integration.

\section{AVALIAÇÃO DE ESTRATÉGIAS EDUCA- CIONAIS DE INTEGRAÇÃO INTERCUL- TURAL}

\section{RESUMO}

Os princípios do Projeto Educacional da Universidade Surcolombiana, propõem a criação de condições que contribuam para uma melhor convivência em um ambiente multicultural. Nesse sentido, o objetivo desta pesquisa é avaliar uma estratégia de integração proposta pelo programa de Educação Infantil, a fim de alcançar um relacionamento mais próximo com cidadãos de outros países e culturas. Por meio de questionários, foram coletadas as percepções dos alunos sobre a relação entre essas atividades e o cumprimento dos princípios institucionais. Os resultados mostram que a atividade que os alunos mais se relacionam com a assimilação e compreensão desses princípios é a dança, enquanto o aprendizado de um seg- undo idioma foi a atividade mais fracamente associada.

PALAVRAS-CHAVE: Multicultural, Interculturalidade, estudantes, integração.

\section{INTRODUCCIÓN}

Los intercambios interétnicos, de nacionalidades, culturas y estratos sociales hacen parte de los retos que enfrenta una educación inserta en un mundo global y por lo tanto multicultural. El gran desafío de la educación es poder conectarse efectivamente para formar equipos competentes. Las relaciones de amistad multinacionales y el aprendizaje, entre otras cosas, de un lenguaje extranjero contribuyen al desarrollo de una cultura universal y una mirada global, (Hendrickson, 2018). El conocimiento de temas universales es también una forma de acercamiento cultural, una vez que dicho elemento es reconocido por los grupos, el descubrimiento de la experiencia y el conocimiento compartido a través de la frontera cultural ayuda a facilitar conversaciones temáticas que deconstruyen la formación de equipos basados en su cultura, (Mori, 2003).

Las migraciones y la mezcla de culturas revelan la importancia de educar interculturalmente (Barreiro et al, 2019). La interculturalidad es un aspecto que sirve de base para comprender la identidad académica y sirve como referencia para una mejora e impacto positivo en su trabajo y entorno profesional (Alonso et al, 2019). A través de un programa de aprendizaje experiencial intercultural, los estudiantes amplían sus esferas culturales reconociendo el esfuerzo para interpretar sus puntos de vista y el de los demás, y las posibilidades de estos aprendizajes para una ciudadanía global intercultural, (Holmes et al, 2016). Desarrollar la conciencia intercultural, apoyada a través de oportunidades para expresar emociones y sentimientos ayuda a los estudiantes a comprender conceptos inter- 
culturales, fundamentales para un pensamiento intercultural (Holmes et al,2015).

Sin embargo, también hay factores que limitan el potencial intercultural; algunos estudiantes internacionales tienen oportunidades limitadas para interactuar con el entorno multicultural y no sienten que están interactuando en igualdad de condiciones con otros estudiantes, (Schweisfurth \& Gu, 2009). Un ejemplo típico es el estatus de inmigrante que provoca en muchos casos el bullying, (Caravitaa et al, 2019). De la misma manera, el estatus social como construcción colectiva influye en la formación de grupos problemáticos tanto en las redes sociales como en la vida cotidiana, reflejándose en agresiones, bullying y otras formas de victimización entre estudiantes, (Rubineaua et al, 2019).

Por otra parte, para que la sociedad sea capaz de vivir en paz, los valores culturales son claves para la integración pedagógica y transformar la escuela en la base de ese escenario (Matos et al, 2019). Ahora bien, bajo ciertas condiciones se pueden crear nuevas redes de líderes para el cambio social, (Matous, 2019). Redes tejidas en diferentes contextos que, según Demaray, (2019), la escuela desde una actitud sicológica debe tener conocimiento de las diferencias individuales, desde este enfoque se aportan herramientas para mejorar el funcionamiento de individuos, familias y escuelas con diversas características, culturales, históricas, atravesadas por múltiples contextos y que pueden, mediante actividades de autoafirmación emocional, mejorar los alcances académicos en estudiantes que hacen parte de minorías étnicas (Lokhande \& Müller, 2019).

Sobre el impacto que generan en la vida y la escuela los prejuicios reduccionistas, los estudios de Brenick et al. (2019), con población árabe-judía, muestran una significativa reducción de prejuicios culturales a partir de interacciones entre los grupos. El sentido de pertenencia, a partir de cruces culturales, puede mejorar los niveles educativos, al tiempo que reducen los niveles depresivos, (Marksteiner et al, 2019).

Se han observado los resultados positivos de hacer cruces grupales para interaccionar con espacios y personas diferentes, a través de ejercicios de escritura creativa, entre estudiantes de diverso origen, (Mäkinena, 2019). A través de la literatura se crea un contenido de infinitas posibilidades para el logro de aprendizajes autónomos, significativos, de enraizamiento cultural desde lo cognitivo, afectivo y creativo. (Chong, 2019). Estas narrativas permiten ver cómo los estudiantes ilustran y reflejan la forma en que reaccionan a su entorno cultural, sus historias y diversos orígenes, brindando un vocabulario que articula las formas en que logran una mejor comprensión intercultural (Giovanangeli \& Oguro, 2016). Así mismo, Harsch \& Poehner (2016) proponen una evaluación dinámica que examine las necesidades de los estudiantes, integrando la enseñanza, aprendizaje y evaluación de los procesos cognitivos desarrollados detrás las narraciones que involucran a individuos de diferentes orígenes lingüísticos.

En general, los resultados positivos de ejercicios periódicos dirigidos para mejorar el comportamiento de los jóvenes están asociados con la permanencia en el tiempo de este tipo de actividades (Binning et al, 2019).

A pesar de lo cerradas, las fronteras físicas se hacen cada vez más flexibles debido a las necesidades económicas y a los saberes que se requieren para establecerse competitivamente en el mundo. En ese mismo sentido, las redes sociales de internet han generado nuevos espacios de convivencia. La intensidad de uso de WeChat está significativamente relacionada con la calidad de las relaciones amistosas, sin embargo, solo se relaciona con niveles de bienestar cuando la calidad de las relaciones es real, y existe un contacto cara a cara (Pang, 2018). Incluso cuando las redes colapsan los grupos migran a otras redes sociales teniendo en cuen- 
ta las relaciones de amistad que se han formado cara a cara, (Lőrincz et al, 2019).

Si bien, en muchas instituciones de educación los cruces culturales son evidentes, no siempre lo es la integración. Teniendo en cuenta todo lo anterior, la Universidad Surcolombiana de Neiva desarrolló una iniciativa enfocada desde el proyecto educativo de la institución, cuyos principios giran alrededor de una mejor convivencia en un ambiente multicultural. En ese orden de ideas, el programa de Educación Infantil ha desarrollado un proyecto trasversal con el fin de que los estudiantes se integren no sólo con los compañeros de las clases sino también con estudiantes o profesionales de otros países y puedan compartir y conocer información de otras culturas, tanto en forma presencial como también utilizando las redes sociales.

Este proyecto se ha realizado en forma transversal durante las clases, pero hasta el momento no ha sido evaluado. Por lo anterior el objetivo de la presente investigación es evaluar una estrategia de integración, a través de la cual se pretende lograr un mejor acercamiento con ciudadanos de otras regiones, países y culturas, para contribuir al cumplimiento de los principios de democracia, epistemología, ética e inclusión que forman parte de los principios en los que se apoya el Proyecto Educativo Universitario, (2016).

\section{PRINCIPIOS DEL PROYECTO EDU- CATIVO UNIVERSITARIO}

El Proyecto educativo Universitario (PEU) recomienda para el desarrollo de los cursos, además de los conocimientos científicos y propios de otras culturas, contemplar también los saberes ancestrales del entorno cultural, procurando un ambiente de negociación intercultural, (Consejo Superior Universitario USCO, 2016). Por lo tanto, mediante la aplicación de los principios del PEU, se debe propiciar la concientización de la comunidad universitaria que está inmersa en un ambiente multicultural, proporcionando un ambiente democrático en cuanto al reconocimiento de las diferencias multiculturales, la ética en cuanto al logro del respeto y la tolerancia, la epistemología en cuanto a la apropiación del conocimiento pertenecientes a las distintas culturas y finalmente la inclusión en cuanto al reconocimiento de la diferencia $u$ otredad.

Comenzaremos con una breve descripción de estos principios (Consejo Superior Universitario USCO, 2016):

Democracia: Ejercicio del poder que reconoce y garantiza las libertades individuales, la igualdad en derechos y oportunidades y las diferencias multiculturales. Por ello, la universidad promoverá la participación deliberativa para el tratamiento de los saberes, garantizando el respeto $y$ la convivencia pacífica en el pluralismo cultural, moral y político, de sus integrantes.

Epistemología: Reconoce la importancia de las condiciones que hacen posible el aumento, la apropiación, transferencia, transformación y producción del conocimiento pertenecientes a las distintas culturas.

Ética: Privilegia una ética cívica de valores que se orientan a lograr unos mínimos básicos de justicia social, respeto entre semejantes, tolerancia, solidaridad entre otros.

Inclusión: Reconocimiento de la diferencia u otredad, como derecho a participar en los procesos formativos sin distingo de color, raza, credo, etnia, género. En general se orienta a la negación de cualquier forma de discriminación. (Consejo Superior Universitario USCO, 2016)

\section{METOdOLOGÍA}

El proyecto que se evaluó se denominó "Cantando y actuando por el saber la paz y el 
medio ambiente" cuyo objetivo fue contribuir al cumplimiento y puesta en práctica de los principios institucionales del proyecto educativo de la Universidad Surcolombiana, (Casadiego et al, 2016).

Los participantes fueron 91 estudiantes del programa Educación Infantil de la Universidad Surcolombiana (USCO), quienes una vez desarrollada la propuesta, dialogaron en diferentes grupos sobre el logro de estas actividades en relación con los principios del PEU, y finalmente, respondieron un cuestionario donde seleccionan las actividades que más perciben adecuadas para lograr los Principios Institucionales propuestos (ver anexo).

La propuesta consistió en tres actividades con las cuales se espera influir tanto en el conocimiento de la otra cultura como en el reconocimiento de que somos multiculturales y debemos vivir en un ambiente de respeto y tolerancia hacia el otro.

Para el desarrollo de los cursos el (Consejo Superior Universitario USCO, 2016), recomienda un prudente balance en el uso de recursos y estrategias didácticas virtuales, con presencialidad docente y de trabajo estudiantil independiente. Por lo tanto, durante el desarrollo del curso, los estudiantes realizan entrevistas virtuales con el visitante extranjero para informarse sobre los platos típicos, bailes y canciones propias de la región. La información recogida es discutida al inicio de la clase de tal forma que todos los estudiantes puedan resolver sus dudas y complementar la información a través del dialogo y el trabajo en equipo.

\section{PRIMERA ACTIVIDAD: IDIOMAS}

Los estudiantes aprenden una canción en el idioma del visitante la cual es cantada al inicio de la clase. Para González (2007), en la mayor parte de las culturas se considera la música como una de las habilidades necesarias para sobrevivir bien y destinado a incrementar el sentimiento de comunidad y establecer un acuerdo con el entorno. Por lo tanto, para contribuir a la conformación de la identidad y lograr que los estudiantes de la USCO sientan un mutuo sentido de pertenencia, además de la canción del país visitante, también se aprenden canciones en inglés con mensajes de paz, como por ejemplo imagine de los Beatles. Así mismo, los estudiantes leen sencillos párrafos en inglés, relacionados con las costumbres y modos de vida del país visitante.

\section{SEGUNDA ACTIVIDAD: DANZAS FOLK- LÓRICAS}

La danza es un arte visual asociado a la música que se desarrolla en el tiempo y en el espacio, está arraigada en las personas y se transmite como legado cultural, Martín (2005). La actividad pretende mediante la danza acercar al estudiante a otras culturas. Los alumnos aprenden una danza del país visitante y es puesta en escena el día de la actividad de integración con el invitado extranjero. Esta actividad implica que en cada clase los alumnos deben establecer acuerdos para el montaje de la coreografía, el vestuario y otros detalles de tal forma que finalmente el resultado sea del agrado de todos.

\section{TERCERA ACTIVIDAD ESTRATEGIA DE COMPARTIR RECETAS}

Para lograr una aproximación cognitiva entre los grupos se va a utilizar la estrategia de compartir recetas. El visitante extranjero enseña una receta típica y a su vez debe aprender otra que los estudiantes le enseñen. Se organizan entrevistas donde se indaga la historia de los alimentos, sus orígenes y los momentos en los que más se acostumbra consumirlos. De igual manera, para aquellos ingredientes de difícil consecución, se discute también la utilización de alimentos opcionales que brinda el entorno. 


\section{RESULTADOS Y DISCUSIÓN}

Los resultados del cuestionario muestran la percepción que tienen los estudiantes, sobre cómo cada una de las actividades realizadas contribuye a la apropiación de los principios del proyecto educativo universitario.

La danza fue calificada como "totalmente de acuerdo" para el desarrollo de actividades que promueven la inclusión o reconocimiento de la diferencia, la epistemología o apropiación y producción conocimiento de distintas culturas y la ética o respeto y tolerancia, acorde con los principios del proyecto educativo.

A su vez, la Receta fue seleccionada como "totalmente de acuerdo" para el aumento de los conocimientos sobre otra cultura.

Finalmente, Idiomas fue seleccionada como "totalmente de acuerdo" para el principio de democracia o ejercicio que reconoce las diferencias multiculturales.

\section{---Insertar tabla 1 aproximadamente aquí---}

En la tabla 1, la danza es la más seleccionada para dar a conocer el mayor número de aspectos del Proyecto Educativo.

En la tabla 2 se encuentra la información anterior discriminada por cada uno de los principios del PEU.

\section{---Insertar tabla 2 aproximadamente aquí---}

De acuerdo a la tabla 2, la danza es la que abarca más posibilidades de relacionarse con los principios del PEU. Estos resultados pueden ser explicados por la forma como se desarrolló la preparación para la jornada de integración, pues en cada clase los alumnos deben establecer acuerdos para la selección de los pasos y figuras del baile, actividad en la que el respeto y la tolerancia deben estar presentes, así mismo es explicable la selección del principio de inclusión, pues el grupo tuvo que tener en cuenta las habilidades y limitaciones individuales para el montaje de la coreografía, resultados que concuerdan con Segura (2016), para quien es posible bailar y respetar las diferencias corporales y reconocerse como territorios plurales e inclusivos.

Por otro lado, la actividad de la receta fue la más relacionada con el aumento de conocimientos sobre otra cultura, es posible que esta percepción se deba al hecho de que en esta actividad se involucran varios procesos de conocimiento en cuanto a que los estudiantes no solo deben indagar sobre la historia de los alimentos, sino que también deben esforzarse en adaptar la receta a los medios provistos por el entorno, además está involucrado el sabor. De acuerdo con Prospéro et al, (2013), la dieta requiere de una serie de procesos cognitivos que se establecen con el aprendizaje.

La actividad de idiomas, en este caso aprender una canción en el idioma del visitante, es la que los estudiantes más relacionan con el principio de democracia, entendida como ejercicio que reconoce derechos y diferencias multiculturales, resultado que se asocia con González (2007), en cuyos trabajos se afirma que en la mayor parte de las culturas se considera la música como una de las habilidades necesarias para incrementar el sentimiento de comunidad y establecer un acuerdo con el entorno. La actividad de idiomas también incluyó la lectura de sencillos párrafos en inglés relacionados con las costumbres del país visitante, lo que puede contribuir también en el reconocimiento de las diferencias multiculturales, según Rico-Martín, (2005), en el aprendizaje de una segunda lengua se reconoce la importancia de la comprensión de costumbres y formas de vida diferentes, 
la tolerancia y el respeto entre los países y sus hablantes. No obstante, esta actividad no fue seleccionada en cuanto al aumento y apropiación de conocimientos de otra cultura, probablemente por el tiempo que se requiere para llegar a ser competente en el dominio de otro idioma.

\section{CONCLUSIONES}

La percepción que tienen los estudiantes en cuanto a la contribución de estas actividades en el acercamiento con ciudadanos de otras regiones y culturas, mediante el cumplimiento de los principios de democracia, epistemología, ética e inclusión que forman parte del PEU, la danza es la más sinérgica pues es la que más relacionan las estudiantes con la asimilación y comprensión de estos principios. La Receta y, paradójicamente, los idiomas fueron las actividades menos asociadas con principios del PEU.

Los principios con los que más se relaciona la danza son la Inclusión, la ética y la epistemología. Es decir, con el respeto, el reconocimiento de las diferencias multiculturales y aunque en menor proporción con el principio epistemológico que hace posible apropiarnos del conocimiento producido por otras culturas y condiciones. Con este último la más relacionada fue la actividad de la receta, mientras que idiomas fue la única relacionada con el reconocimiento de las diferencias multiculturales es decir con el principio de democracia.

Este resultado es de suma importancia a la hora de implementar programas con el fin de lograr mayor pertenencia a un ambiente multicultural, pues implica tener en cuenta un proceso de aprendizaje que debe mantenerse a través del tiempo. El aprendizaje de las recetas se podría complementar con un conocimiento más profundo alrededor de la cultura y los rituales alrededor de la comida. Por su parte, el aprendizaje de los idiomas es exitoso en la me- dida en que este se vuelva parte de la cotidianidad. En una actividad transversal a una actividad de clase, muy difícilmente se logra este nivel de inmersión. La danza por el contrario es un tránsito más fácil hacia el conocimiento y en el caso de las estudiantes de Educación Infantil, se encuentra entre las asignaturas que forman parte del programa, por lo cual ya existe una familiaridad más cercana a este tipo de actividades que involucran el cuerpo y el movimiento.

Sería interesante evaluar esta estrategia en un curso de idiomas, de tal manera que podamos observar las percepciones de estudiantes en los que el idioma ya no es una barrera intercultural.

\section{REFERENCIAS}

Alonso-Saéz, I., Garagarza, A., Karmele A. y Nekane, B. (2019) Bases para un modelo de guía para la transformación de las identidades docentes universitarias, Boletín Redipe, 8(8), 68-82

Barreiro, M. P. R., Velásquez, B. I. H., Lara, D. L. L., \& Villavicencio, M. M. P. (2019). Actitudes de estudiantes universitarios hacia la educación inclusiva. Revista Boletín Redipe, 8(9), 89-99.

Binning, K. R., Cook, J. E., Purdie-Greenaway, V., Garcia, J., Chen, S., Apfel, N., ... \& Cohen, G. L. (2019). Bolstering trust and reducing discipline incidents at a diverse middle school: How self-affirmation affects behavioral conduct during the transition to adolescence. Journal of school psychology, 75, 74-88.

Brenick, A., Lawrence, S. E., Carvalheiro, D., \& Berger, R. (2019). Teaching tolerance or acting tolerant? Evaluating skills-and contact-based prejudice reduction interventions among Palestinian-Israeli and Jewish-Israeli youth. Journal of school psychology, 75, 8-26. 
Casadiego A,Trujillo C,Chávarro G,Cuervo C y Díaz L (2016). Cantando y actuando por el saber, la paz y el medioambiente. Colombia: Ed:Alix Casadiego

Caravita, S. C., Strohmeier, D., Salmivalli, C., \& Di Blasio, P. (2019). Bullying immigrant versus non-immigrant peers: moral disengagement and participant roles. Journal of school psychology, 75, 119-133.

Consejo Superior Universitario USCO (2016) , Acuerdo 010 de 2016 Proyecto Educativo Universitario, Universidad Surcolombiana, Neiva, Colombia.

Chong, B. M. F. (2019). Educar desde la literatura: enraizamiento cultural para una pedagogía comprensiva. Revista Boletín Redipe, 8(8), 83-94.

Demaray, Michelle K. (2019). Editor.Acculturation and school adjustment of children and youth from culturally diverse backgrounds: Predictors and interventions for school psychology. Journal of School Psychology. 75 1-7

Giovanangeli, A., \& Oguro, S. (2016). Cultural Responsiveness: a framework for re-thinking students' interculturality through study abroad. Intercultural Education, 27(1), 70-84. https://doi.org/10.1 080/14675986.2016.1144328

González, P. F. (2007). La formación musical como pasión comunicativa. Educação, 30(62), 287-298.

Harsch, C., \& Poehner, M. E. (2016). Enhancing student experiences abroad: the potential of dynamic assessment to develop student interculturality. Language and Intercultural communication, 16(3), 470490. https://doi.org/10.1080/14708477.2 016.1168043
Hendrickson, B. (2018). Intercultural connectors: Explaining the influence of extra-curricular activities and tutor programs on international student friendship network development. International Journal of Intercultural Relations, 63, 1-16.

Holmes, P., Bavieri, L., \& Ganassin, S. (2015). Developing intercultural understanding for study abroad: students' and teachers' perspectives on pre-departure intercultural learning. Intercultural Education, 26(1), 16-30. https://doi.org/10.1080/14 675986.2015.993250_

Holmes, P., Bavieri, L., Ganassin, S., \& Murphy, J. (2016). Interculturality and the study abroad experience: Students' learning from the IEREST materials. Language and Intercultural Communication, 16(3), 452-469. https://doi.org/10.1080/14708 477.2016.1168054.

Pang, H. (2018). WeChat use is significantly correlated with college students' quality of friendships but not with perceived well-being. Heliyon, 4(11), e00967.

Lokhande, M., \& Müller, T. (2019). Double jeopardy-Double remedy? The effectiveness of self-affirmation for improving doubly disadvantaged students' mathematical performance. Journal of school psychology, 75, 58-73.

Lőrincz, L., Koltai, J., Győr, A. F., \& Takács, K. (2019). Collapse of an online social network: Burning social capital to create it?. Social Networks, 57, 43-53.

Matos, S. L., Martínez, A. R., \& López, J. M. T. (2019). Valores educativos comunes y específicos: análisis descriptivo de su integración pedagógica en las materias escolares a partir de la percepción de los docentes sobre su actividad. Revista Boletín Redipe, 8(6), 23-49. 
Mäkinen, V., Liebkind, K., Jasinskaja-Lahti, I., \& Renvik, T. A. (2019). A teacher-led vicarious contact intervention in culturally mixed classrooms with in-and outgroup role models of intergroup friendship. Journal of school psychology, 75, 27-40.

Marksteiner, T., Janke, S., \& Dickhäuser, O. (2019). Effects of a brief psychological intervention on students' sense of belonging and educational outcomes: The role of students' migration and educational background. Journal of school psychology, 75, 41-57.

Martín M.J. (2005). Del movimiento a la danza en la educación musical. Educatio siglo XXI, (23), 125-138

Matous, P., \& Wang, P. (2019). External exposure, boundary-spanning, and opinion leadership in remote communities: a network experiment. Social Networks, 56, $10-22$

Mori, J. (2003). The construction of interculturality: A study of initial encounters between Japanese and American students. Research on language and social interaction, 36(2), 143-184. https://doi. org/10.1207/S15327973RLSI3602_3

Prospéro-García, O., Méndez Díaz, M., Capuleño, I. A., Morales, M. P., Juárez, J. L., \& Ruiz Contreras, A. E. (2013). Inteligencia para la alimentación, alimentación para la inteligencia. Salud mental, 36(2), 101-107.

Rico-Martín, A. M. (2005). De la competencia intercultural en la adquisición de una segunda lengua o lengua extranjera: conceptos, metodología y revisión de métodos. Porta Linguarum (3), 79-94 jection: How status hierarchies influence negative tie formation. Social Networks. 56 (33-44)

Segura, C. C. (2016). Danzando en la diversidad: reflexiones sobre la inclusión y pertinencia de la diversidad en la educación dancística y artística. Praxis Pedagógica, 16(18), 27-51.

Schweisfurth, M., \& Gu, Q. (2009). Exploring the experiences of international students in UK higher education: possibilities and limits of interculturality in university life. Intercultural Education, 20(5), 463-473. https:// doi.org/10.1080/14675980903371332

Rubineaua, Limb, Neblo. (2019). Low status re- 
ANEXO

\section{INSTRUMENTO DE EVALUACIÓN DE LA ACTIVIDAD DE INTEGRACIÓN}

\section{Nombre}

Código

De acuerdo a la siguiente escala de 1 a 5

1. En total desacuerdo

2. En desacuerdo

3. Ni de acuerdo ni en desacuerdo

4. En acuerdo

5. Totalmente de acuerdo

Por favor marque el grado de acuerdo o desacuerdo de la relación entre la actividad de integración "receta, danzas e idiomas" con los principios y aspectos del Proyecto Educativo Universitario (PEU):

\begin{tabular}{|c|c|c|c|}
\hline \multirow{2}{*}{$\begin{array}{l}\text { Etica: valores que se orientan a lograr unos mínimos } \\
\text { básicos de justicia social, respeto entre semejantes, toler- } \\
\text { ancia y solidaridad. }\end{array}$} & Receta & Danzas & Idiomas \\
\hline & & & \\
\hline \multicolumn{4}{|l|}{$\begin{array}{l}\text { Democracia: Reconocer la igualdad en derechos y oportu- } \\
\text { nidades y las diferencias multiculturales }\end{array}$} \\
\hline \multicolumn{4}{|l|}{ Epistemología: aumento del conocimiento de otra cultura } \\
\hline \multirow{2}{*}{\multicolumn{4}{|c|}{$\begin{array}{l}\text { Epistemología: apropiación y producción de conocimiento } \\
\text { perteneciente a distintas culturas. }\end{array}$}} \\
\hline & & & \\
\hline
\end{tabular}

